\title{
Research on the Incentive Mechanism of Civil Servants' Human Resources
}

\author{
Sun Haiting 1 ,a

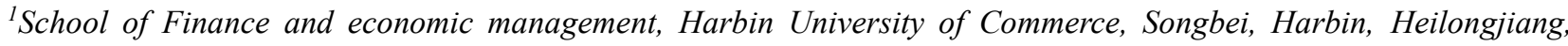 \\ China \\ a962791137@qq.com
}

\begin{abstract}
With the development of new public management theory, the functions of the government are constantly changing, and the masses of the society have more and more requirements and expectations for the government. As the users of government power, civil servants have an irreplaceable role and status, which is also a challenge to the quality of civil servants. How can we improve the enthusiasm of civil servants, work efficiency, loyalty and sense of belonging, which requires a series of incentive mechanisms to achieve the corresponding effect. This paper mainly studies the incentive mechanism of civil servants' human resources to provide new ideas for the construction of civil servants' human resources incentive.
\end{abstract}

Keywords: Civil servants, human resources, incentive mechanism, public management theory.

\section{公务员人力资源激励机制研究}

\author{
孙海婷 ${ }^{1,}$ a \\ ${ }^{1}$ 哈尔滨商业大学财政与经济管理学院, 松北, 哈尔滨, 黑龙江, 中国 \\ a962791137@qq.com
}

\section{摘要}

随着新的公共管理理论的发展，政府的职能在不断转变，社会广大群众对于政府的要求也越来越多，期望也 越来越高。公务员作为政府权力的使用者具有不可替代的作用和地位，这也是对公务员素质的一个挑战。怎 么样才能够让公务员的工作积极性得以提高, 提升工作效率, 增强忠诚度和归属感, 这就需要一系列的激励 机制来达到相应效果。本文主要是对公务员人力资源激励机制进行研究，给公务员人力资源激励构建提供新 的思路。

关键词: 政府职能，人力资源，激励机制，公共管理理论

\section{1. 前言}

公务员是依法行使国家行政权力、执行国家公 务的人员, 从某种意义上讲就是为人民服务的公 仆, 是国家或社会的代理人, 由国家或社会委托来 从事社会公共事务, 受国家或社会人民的监督。对 于公务员的管理与激励是提高政府效率、转变政府 职能的关键, 也是公务员制度自身的需要。[1]激励 是人力资源的核心内容之一, 它能够使员工工作积 极性得到快速提升, 让组织能够获得更加好的发 展。所以, 采用科学的激励措施, 能够提升公务人
员的忠诚度，使公务员的主动性、创造性也获得更 好的提升 [2]。同时，作风的转变也能提高公务人员 的办事效率，让公务人员队伍能够更加的勤政务 实，提升政府的形象，从而促使政府绩效的提高。

\section{2. 公务员人力资源激励的内容与特征}

\section{1. 公务员人力资源激励的主要内容}

我国公务员人力资源激励的内容非常丰富，大 体来说是由五个方面组成的，并且这五个方面之间 
是相互联系和制约的，包括考核、薪酬、奖惩、培 训、晋升体系。他们的具体内容如下：（1）考核方 面。公务员的考核制度, 制定了科学的考核方法以 及考核标准，能够全方位的进行公务员考核，考核 结果关系到该公务员的福利、薪酬、任免等，甚至 还关系到未来的晋升等多个方面 [3]；（2）薪酬方 面。薪酬制度也是最普遍的激励手段。薪酬制度构 建的是否合理, 实施的是否顺利非常重要, 能够保 障公务员对工作的认真负责程度, 维系着其对于工 作的积极性, 因此也是提高公务员效率的重要内容 之一; (3) 奖惩方面。奖惩是除了薪酬之外另一个 常见的激励手段, 合理、公正的奖励, 能够让公务 员自身的价值得到认可, 使得其能够充分的发挥潜 力, 提高能力、端正工作态度, 使得工作效率和质 量有很大的提升，通过工作也是其自身价值的一种 体现, 更能够为政府部门完成工作目标做出更大贡 献;（4）培训方面。公务员激励体制中有着非常全 面的培训体系, 我国的公务员一旦入职就享有终身 职业教育的权利。主要包括新成员的入职培训、晋 升人员的任职培训、专业的技能培训、各类知识的 更新等等; (5) 晋升方面。公务员的激励机制里, 晋升制度的激励作用起着很大的作用。晋升直接表 明公务员自身的工作能力, 能够激发其工作热情, 对于吸引和留住公职人员具有很重大的意义。公平 的晋升制度使得公务员的工作作为示范能够升迁的 标准, 有助于公务员团队内部形成奋发向上的动 力, 让大家积极的形成良性竞争, 通过业绩来获得 晋升机会。其次, 通过晋升机制能够将内部人才进 行再次分配, 实现高效的人力资源管理, 最终达到 提升效率的目的。

\section{2. 公务员人力资源激励的主要特征}

公务员既是制定国家政策、方针、法律的参与 者, 同时也是执行者, 并且还肩负着管理社会的责 任 [4]。由此可知, 公务人员的工作能力和行政效力 与公务人员自身素质、工作能力、工作状态等有着 很大的关系。所以, 要想进一步提升工作的效率, 就应该在人力资源上采取一定的措施, 在对公务员 进行招聘、培训时, 需要重视公务员的道德和政治 素质, 确保有关人员在职业素养、道德、思想政治 观念等各方面水平上都有更好的表现。首先, 不断 提高的客观要求和自身价值表达。当前, 随着信息 技术快速的发展，全球的经济也被不断的联合到了 一起, 人类社会也进入了一个全新的知识经济时 代, 国际和国家的各类事物也变得更加的复杂, 这 些变化使得对公务员要求的不断提高 [5]。其次, 在 进行人力资源的管理过程中, 政府机关应该加大对 公务人员素质培养和提升的力度, 通过不断的开 发、激励等措施, 确保公务人员的自身价值能够跟 上社会发展的脚步。

\section{3. 公务员人力资源激励的必要性}

\section{1. 有利于提高行政效率}

激励机制在公务员制度中扮演着重要的角色, 一 般由考核、晋升、奖励、工资福利、社会保险和培 训等制度构成 [6]。有竞争就有活力, 完备的激励机 制一定蕴含着竞争。在我国的公务员制度中就缺少 了这种由竞争带来的作用, 这也是导致我国公务员 激励机制中行政效率不高的因素之一，因此在公务 员激励机制中注入竞争是非常有必要的, 恰当的竞 争会激发出公务员的积极性，从而激发出他们的工 作潜能, 使公务员在为人民服务的同时锻炼自我突破 自我实现自我, 达到自我价值与社会价值的双赢。

\section{2. 有利于勤政廉政建设}

对公务员考核其实是对其思想、工作的一种考 核和评价。这种考核和评价对公务员的晋升起着很 大的作用, 如果考核不规范则制度形同虚设。对于 公务员的考核内容有很多, 主要是检查公务员的行 为是否符合规范, 是否尽职尽责为人民服务, 是否尽 到了人民公仆应尽的责任。这种考核合评价其实是 对公务员的一种监督和督促, 使其行使权力时考虑各 方因素从而真正的为人民服务, 把自己塑造成更优 秀的公仆形象。此外, 还可以通过考核的方式对公 务员评出不同的等级, 不同的等级对公务员的晋升、 奖励、工资福利等会产生重要的影响。对于考核结 果较差的公务员会激发其向上的动力, 对于考核结 果较好的公务员则会竞争压力, 迫使其更加努力工 作, 使社会形成鼓励先进鞭策后进的良好氛围。

\section{3. 有利公务员队伍建设}

当前政府改革的目标是把政府塑造成服务型政 府, 因此对于公务员要奉行的宗旨就是全心全意为人 民服务。[7]为人民服务不是说说而已，而是要落实 到实处。要想建设高素质队伍就要从制度根源解 决, 通过完善晋升制度, 加强对公务员的考核, 对 于考核结果较好的人员可以进入领导层次, 对于考核 结果较差的公务员进行惩罚, 奖励先进, 惩罚后进, 使得公务员队伍层次越来越合理, 素质越来越高, 通过对制度根源的改革, 有利于公务员队伍建设。

\section{4. 公务员人力资源激励存在的问题}

\section{1. 激励理念相对陈旧}

数千年的发展一直影响着我国公务人员人力资 源管理的机制。传统 “官本位” 的思想使得大多数 公务员把心思放在职位的晋升方面，而没有发挥人 民公仆的示范效应，成为了 “形式上” 的公务员。 同时由于这数千年的封建思想, 在激励的方式上没 有过多的创新, 通常仅仅在精神上进行激励, 忽视 
了对公务人员的物质激励 [8]。很明显在当前的经济 状况下，仅仅的精神激励已经无法满足公务员的日 益复杂的需求，所以在完善公务员激励机制的过程 中，应该改变传统落后的思想，增加全新的激励措 施，借鉴国外先进的经验，取其精华去其糟粕，采 用多元化的模式使公务员保持更好的状态，确保公 务员充分发挥其最大的作用。

\section{2. 人才流动机制價化}

在传统观念和体制的影响下，我国的公务员人 力资源流动化程度比较低, 首先表现在流动机制比 较僵化, 通俗的解释是公务员队伍进去容易退出 难, 在横向流动上缺乏合理的进出规则; 第二是增 项的僵化, 就是没有合理的升迁规则, 通俗来说就 是只能上升而不能下降。这种人才流动机制的偪化 严重影响我国行政的发展, 对于这种问题应从公务 员体制改革入手，把体制改革作为全面深化改革的 突破口, 改革最难改的地方, 改革最顽固的地方, 改革最传统的地方, 破除身份地位间的界限, 最终 出激发制度活力。

\section{3. 晋升激励制度不健全}

晋升制度可以提高公务员工作积极性, 也可以让 他们他们发现自我价值, 实现自我价值。可从晋升 方面来看, 公平晋升的力度存在一些问题, 首先表 现在某些地方政府对干部的任职要求过分强调 “德” 的表现, 而忽视其在生活中的实际表现。其 次是任人唯亲的现象仍大量存在。在我们生活中, 血 缘关系是最坚实、最稳定的利益共同体，基于血缘 关系的权利直接成了破坏行政激励机制, 成为进一步 影响行政效率的重要因素。[9]行政效率低下严重影 响政府形象, 导致公众与政府关系恶化。而在公务 员队伍中职位较低的人员中, 由于职位层次很少, 竞 争的人却很多, 形成人多粥少的局面, 很多人争取有 限的职位, 出现内卷躺平等不良现象, 这种恶性竞争 也是导致行政效率低下的原因之一, 随之而来导致 其失去对于努力工作的兴趣和热情。

\section{5. 公务员人力资源激励问题解决措施}

\section{1. 努力更新激励理念}

第一, 要树立以人为本的理念。这里所说的 “以人为本”, 就是将人力资源放在首位, 借鉴企业 中人力资源管理的思想, 在公务员队伍中融入公共 部门人力资源管理制度。[10]这最重要的就是就是 把公务员自身的追求与政府的工作目标有机结合起 来, 通过采取一系列的措施, 使公务员在实现自我价 值的同时也实现服务于社会公众的目标。对于公务 员的激励要采取灵活科学的教育方式, 不能一刀 切, 更不能只是做做样子。通过科学的方式引导公 务员树立责任感, 激发出其对工作的热情, 使其真
正热爱这份工作，帮助公务员树立正确的服务观 念, 端正服务态度, 克服争权夺利、争权夺势的现 象, 以真正热爱为公务员工作的内部动力。对此我 们要创造令其满意的工作环境, 同时根据自身情况 制定自身的发展方向, 使公务员清晰的认识个人发展 目标并实现自身发展需求, 挖掘出公务员的个人价 值。在对公务人员进行激励的同时要注意采用与时 俱进的方法, 不能始终依照传统的方式进行激励, 要将以人为本的激励理念作为基础, 重视公务人员 的基本需求, 并且针对不同层次的需求, 选择不同 的激励措施, 深刻意识到人力资源的重要意义, 在 培训和教育上也加大重视力度; 同时, 要树立法制 化观念。根据宪法的要求以及公务人员的具体工作 内容, 不断的建立和完善法律体系, 真正使激励体 制更加的法制化、科学化、人性化。同时, 坚决对 违法行为进行纠正, 依法追究相关人员的法律责 任, 以此来确保激励的合法进行。

\section{2. 完善人才动态管理}

首先，对竞争淘汰机制进行完善，建立末位淘 汰制, 通过竞争的形式上岗, 增强公务人员工作的 紧迫性和积极性, 让公务人员加大力度投入到工作 之中。对于工作能力较差、业绩不足的公务人员要 进行脱岗培训, 通过适当的形式来增强其办事能 力, 进而能够胜任本岗位的工作。试岗期间暂停奖 金发放，并且领导对试岗人员进行一对一的帮扶。 试岗期过后, 才能够进行正式的工作安排; 对于试 岗不合格的人员, 需要根据规定进行辞退或者解 聘。通过一系列完整的淘汰机制, 加强公务员危机 感, 进而提升工作水平和动力。其次, 要构建灵活 的人才晋升机制。晋升对于公务员来说是非常重要 的, 是一种非常有效的激励形式, 既具有精神效果 又有物质效果, 通过晋升可以加强公务员对其工作 的热爱，同时完善的制度也会给与制度奖励。科学 的构造人才晋升机制, 有利于提高公务人员的积极 性、主动性和创造性。为此我们需要对人才晋升机 制进行改革, 主要要实现以下三点: 第一、晋升的 主要依据是绩效, 通过科学的晋升步骤, 通过竞争 上岗制度和职位任期制度, 在制度层面上制止官官 相护现象出现, 也避免了恶性竞争情况的发生, 使 公务员晋升的越来越科学、越来越合理。第二, 通 过制定规范且明确的标准, 逐步加强晋升的制度化 和法制化，在晋升的监督上制定完善的体制，让公 务员在完全透明的情况下了解晋升的全过程。第 三, 要加强对年轻公务员的培养, 对于工作能力较 强的年轻公务员提供更多的发展空间, 让其感受到 自身的价值，进而做到人尽其才、才尽其用。通过 对人才晋升机制的改革, 完善人才动态管理。

\section{3. 完善激励制度}

要完善晋升制度, 首先应该将晋升与绩效考核有 机的结合起来。将晋升的各种程序步骤全部公开, 让 
每个公务员都了解晋升的程序。同时, 通过在晋升中 增加考试的办法使结果更加客观, 增加考试并不是完 全依赖考试，而是通过考试的方式使结果更加客 观。其次, 要加强对晋升负责人的监督, 把责任落实 到具体个人上，同时加强对其工作程序与方法等的 监督, 使其工作公开透明, 避免发生贪污受贿等腐败 徇私舞弊等现象的发生。同时应加强与纪检部门的 合作, 使整个晋升过程更得到监督。最后, 将公务员 职务晋升信息通过媒体透明化, 对公众反映加以重 视，使公务员晋升制度在阳光之下。相信通过对于 晋升制度的完善, 可以使公务员对激励机制产生足够 的信任感, 促进激励机制度的有效运行。

\section{6. 结论}

随着我国政治体制改革的深入发展，公务员更 需要积极合理的行使国家赋予的权利来服务于社会, 满足人民大众的要求和期望。但是, 目前从我国公务 员队伍的实际情况来看, 虽然我国公务员制度与国外 发达的公务员管理制度相比还有较大的差距，一些 地方还有制度上的缺陷，但通过这些年的改革和完 善已经逐渐成为一套较为完备的体系。在我国公务 员制度中激励制度是重要的组成部分, 我们要充分认 识到它的缺陷与不足, 相信通过不断地总结经验弥 补不足, 根据制度在实际运行中的具体情况, 最终 建立起具有中国特色的公务员管理制度.

\section{REFERENCES}

[1] Yanhua, T., (2017) Inspiration of foreign incentive mechanism for grassroots civil servants to China -- Taking the United States, Japan and Vietnam as examples. J. Explore., 06: 187-191.

[2] Jianping, C.,Zhen, C., (2017) Analysis on the cognition of grassroots civil servants' incentive expectation under the background of the reform of the merger system. J. Administrative reform., 06: $48-51$.
[3] Yulian, Y.,Guoqing, Z., (2017) Game analysis of mediocre officials and lazy government. J/OL. ournal of Zhejiang University (HUMANITIES AND SOCIAL SCIENCES EDITION)., 47 (02) : 138-147.

[4] Lijuan, D., (2015) Analysis on the dilemma and Countermeasures of the reward system for civil servants in China. J. Hunan Social Sciences., 02: $47-51$.

[5] Zhen, C., (2016) On perfecting the incentive mechanism of civil servants. J. Journal of Gansu Administration College., 03: 15-17.

[6] Ju, Z., (2016) Research on the problems and Countermeasures of incentive mechanism for civil servants in China. J. Managing the world., 04: $170-171$

[7] Lei, D., Xiao, W., Jiapeng, L., (2018) On the derivative problems of the merger of local tax institutions in China. J. Tax and economy., 06: 94-99.

[8] Yun, F., Chunxiao, L., Keqin, Z., (2018) The influence of reward and punishment standards on employee silence: the mediating role of perceived safety and trust. J. East China Economic Management., 06: 133-140.

[9] Hongxing, Z., (2017) Research on the application of enterprise incentive mechanism in human resource management of public sector. J. knowledge economy., 17: 85-86.

[10] Cheng, Z., (2018) The construction and improvement of the positive incentive mechanism for cadres J. Learning theory., 17: 75-76. 